Universidad y Salud

ARTÍCULO DE REVISIÓN

\title{
Violencia de género: Un análisis evolutivo del concepto
}

\author{
Gender violence: an evolutionary analysis of the concept
}

Cruz Deicy Jaramillo-Bolívar1,2,3* orcid.org/0000-0003-0945-3279

Gladys Eugenia Canaval-Erazo' ${ }^{1}$ orcid.org/0000-0001-9841-5084

\author{
1 Grupo de investigación PROMESA, Universidad del Valle, Cali, Colombia. \\ 2 Programa de Enfermería, Universidad Libre seccional Cali, Colombia \\ 3 Grupo de investigación Atención primaria y políticas públicas, Universidad Libre seccional Cali, Colombia
}

Fecha de recepción: Octubre 18 - 2018

Fecha de revisión: Marzo 15 - 2019

Fecha de aceptación: Abril 30 - 2020

Jaramillo-Bolívar CD, Canaval-Erazo GE. Violencia de género: Un análisis evolutivo del concepto. Univ. Salud. 2020;22(2):178-185. DOI: https://doi.org/10.22267/rus.202202.189

\section{Resumen}

Introducción: Un concepto es una idea acerca de algo o de una acción. Objetivo: Analizar el concepto "Violencia de Género" desde la perspectiva evolutiva de Rodgers. Materiales y métodos: Se utilizó el análisis evolutivo de conceptos mediante revisión de literatura publicada entre los años 2000 y 2018. Criterios de inclusión: Artículos de investigación, de implementación de estrategias, de política, tesis, libros, capítulos de libro y reportes, en las bases de datos Lilacs, Ebsco, SciELO, CINAHL, Web of Science, PubMed, Google Académico; también se incluyeron documentos de organizaciones internacionales, publicados del año 2000 al año 2018, en Español, Inglés o Portugués, y que pertenecieran al contexto iberoamericano. Resultados: La Violencia de género consiste en un fenómeno de orden estructural, social y político; resultado de la discriminación estructural originado por un sistema sexo-género-patriarcado. Se encontraron términos relacionados como inequidad de género, control, relaciones asimétricas y términos subrogados como violencia de pareja, contra la mujer y doméstica. Conclusiones: La violencia de género, constituye una violación a los derechos humanos, afecta la dignidad, la integridad física y moral, la libertad y la autonomía.

Palabras clave: Violencia de género; violencia contra la mujer; análisis de concepto. (Fuente: DeCS, Bireme).

\section{Abstract}

Introduction: A concept is an idea about something or an action. Objective: To analyze the concept of "Gender Violence" from the Rodgers' evolutionary perspective. Materials and methods: An evolutionary analysis of this concept was carried out through a review of literature published between 2000 and 2018. The inclusion criteria were: research articles related to implementation of strategies and politics; thesis; books; book chapters; and reports. They were found in the databases of Lilacs, Ebsco, SciELO, CINAHL, Web of Science, PubMed, and Google Scholar. The review also included documents from international organizations published from 2000 to 2018 in Spanish, English or Portuguese, which discussed Ibero-American issues. Results: Gender violence is a phenomenon with structural, social and political dimensions and is caused by the structural discrimination triggered by a sexgender-patriarchy system. Related terms such as gender inequality, control, asymmetric relationships were found. Likewise, surrogate terms, i.e., violence against intimate partners, women and domestic violence, were also identified. Conclusions: Gender violence constitutes a violation against human rights that affects the dignity, physical and moral integrity, freedom, and autonomy of people.

Keywords: Gender based violence; violence against women; concept analysis. (Source: DeCS, Bireme).

\footnotetext{
*Autor de correspondencia

Cruz Deicy Jaramillo B

e-mail: cruz.jaramillo@correounivalle.edu.co
} 


\section{Introducción}

Un concepto es la imagen mental de un fenómeno, es una idea o un constructo en la mente acerca de algo o de una acción; es una idea general, abstracta e inarticulada que sirve de marco para la construcción de argumentos que permiten caracterizar el elemento central para el desarrollo de una teoría(1).

Los conceptos son expresados por medio del lenguaje; contienen dentro de sí mismos los atributos o características que los hacen únicos y diferentes de otros conceptos y permiten definir, describir y explicar fenómenos(2).

Este estudio de investigación secundaria se realizó con base en la revisión de la literatura publicada en un período que abarcó los últimos 18 años; bajo el marco de la teoría de desarrollo de conceptos mediante la técnica de análisis conceptual que permite dar significado y definir conceptos que, por ser usados de manera amplia, se tornan con el paso del tiempo en vagos o ambiguos.

El análisis evolutivo del concepto de violencia de género facilitará su comprensión y uso en los diferentes campos del saber, dado que este concepto se usa de manera equivalente con el de violencia basada en el género y se relaciona con el de violencia contra la mujer.

Su evolución a partir de la década del 70 del siglo pasado parte del término acuñado como violencia contra la mujer por organismos rectores en salud y en derechos humano tales como la Organización Mundial de la Salud, las Naciones Unidas y de documentos como la Convención de Belén do Pará que dieron directrices para la legislación de muchos países entre ellos Colombia.

En Colombia, la Ley 1257 de 2008 emplea el término violencia contra la mujer; la define como "cualquier acción u omisión, que le cause muerte, daño o sufrimiento físico, sexual, psicológico, económico o patrimonial por su condición de mujer, así como las amenazas de tales actos, la coacción o la privación arbitraria de la libertad, bien sea que se presente en el ámbito público o en el privado" (artículo 2 de 2008)(3). Los Lineamientos de la Política Pública Nacional de Equidad de Género para las Mujeres en el año 2012 emplean el término violencia basada en el género. Esta violencia que ocurre contra las mujeres por el hecho de ser mujeres se define como violencia basada en género(4).

Comprender el concepto de violencia de género y reconocer sus características o atributos permite establecer claridad conceptual para su uso en la investigación y en la práctica en diferentes campos; además permite diferenciarlo de otros términos que se van tornando imprecisos y se usan en el lenguaje común, lo que conduce a la representación de situaciones que no corresponden a la realidad(2,5).

Esta evolución del concepto de violencia de género se presenta como "una manifestación más de la resistencia que existe a reconocer que la violencia contra las mujeres no es una cuestión biológica, ni doméstica sino de género", lo señala María Luisa Maqueda en su artículo: La violencia de género: Entre el concepto jurídico y la realidad social (pág. 2) en el que señala que "Es significativo que hasta muy avanzado el siglo pasado no se encuentre ninguna referencia precisa a esa forma específica de violencia en los textos internacionales, salvo acaso como expresión indeterminada de una de las formas de discriminación contra la mujer proscrita por la Convención de Naciones Unidas de 1991"(6). A partir de los años noventa, comienza a consolidarse su empleo gracias a iniciativas como la Conferencia Mundial para los Derechos Humanos celebrada en Viena en 1993, la Declaración de Naciones Unidas sobre la eliminación de la violencia contra la mujer del mismo año, la Convención Interamericana para prevenir, sancionar y erradicar la violencia contra la mujer (1994) o la Conferencia Mundial de Mujeres de Beijing (1995)(6).

Para el análisis de conceptos existen diferentes propuestas, una es la planteada por Walker y Avant, otra es la de Rodgers, otra es la de Wilson, y otra es la de Koort(1,7). El marco teórico sobre desarrollo de conceptos muestra que éste se realiza a través de una de tres aproximaciones, una es el "análisis del concepto" cuyo propósito es aclarar, refinar y dar forma al concepto; otra manera es la denominada "síntesis" usada para construir nuevos conceptos y la tercera, es la "derivación" que redefine un concepto de un contexto a otro con el empleo de analogías y metáforas ${ }^{(1)}$. Por lo tanto, el objetivo de este estudio es analizar el concepto de violencia de género a partir de la revisión de la literatura, centrado en el uso y evolución del concepto desde la perspectiva evolutiva de Rodgers. 


\section{Materiales y métodos}

En el análisis evolutivo del concepto propuesto por Rodgers se empleó el método sistemático e inductivo que parte de la elección del concepto, delimitar el contexto del concepto y realizar la recolección de los datos para el análisis en textos, artículos, libros, informes, etc.(7). El método incluye definir el concepto, sus términos subrogados o substitutos, los relacionados, los antecedentes y consecuencias, el identificar si es necesario, un ejemplo del concepto y determinar sus implicaciones ${ }^{(8)}$.

Los componentes centrales que guiaron la recolección de los datos son los antecedentes, los atributos y las consecuencias, también los términos subrogados y los relacionados. El proceso de análisis fue riguroso, incluyó la revisión y la síntesis de las fuentes de la literatura lo que permitió diferenciar un concepto de otro relacionado o similar. El resultado es siempre considerado provisional; a pesar de llegar a identificar atributos o a definir el concepto elegido, se presentan en el futuro cambios en sus atributos por la evolución del mismo, a lo largo del tiempo. Un concepto definido en un momento dado podría no ser igual en el futuro.

Los antecedentes son eventos o fenómenos que previamente han sido relacionados con el concepto y que responden a la pregunta, ¿Qué ocurrió previamente en relación con el concepto? La respuesta puede ser una emoción, una experiencia, o un tipo de comunicación.

Las consecuencias son el resultado del uso del concepto en una situación práctica, los términos sustitutos son los diversos términos, palabras o expresiones utilizadas con el mismo sentido y los conceptos relacionados corresponden a suposiciones filosóficas y a una red de otros conceptos que permiten la formulación de un significado para el fenómeno estudiado.

Los atributos son el conjunto de características físicas o sociales o procesos relacionales que hacen posible identificar situaciones que se categorizan bajo el concepto en estudio. Los atributos pueden cambiar a medida que la comprensión del concepto mejora o cuando se utiliza en un contexto diferente al que se estudia.
En la elección se observa de qué manera se ha utilizado en las diferentes áreas del conocimiento; se tuvo en cuenta la relevancia para la práctica, la definición y la ambigüedad en cuanto a su significado o uso dentro del contexto de la práctica en las ciencias de la salud.

Procedimiento. Se determinaron como criterios de inclusión: Artículos de investigación, de implementación de estrategias, de política, tesis, libros, capítulos de libro y reportes, en las bases de datos Lilacs, Ebsco, SciELO, Cinahl, Web of Science, PubMed, Google Académico; también se incluyeron documentos de organizaciones como la Organización Mundial de la Salud, la Organización de Naciones Unidas y la Comisión Económica para América Latina (CEPAL), publicados en el rango del año 2000 al año 2018, en idioma Español, Inglés o Portugués, disponibles en texto completo y que pertenecieran al contexto iberoamericano. Se emplearon como palabras claves violencia de género, violencia contra la mujer y análisis de concepto de violencia de género.

\section{Resultados}

La muestra final comprendió 43 estudios de los cuales 16 fueron publicados en España, 15 en Colombia, 5 en México, 5 en Brasil, uno en 1 Cuba y uno en 1 Uruguay. Los trabajos seleccionados corresponden al período 2000 al 2018 la mayoría de ellos en la década de los años 2010 en adelante

En la tabla 1 se presentan los elementos del análisis conceptual y el número correspondiente del estudio.

\section{Contexto del concepto}

El concepto de violencia de género está vinculado a un sistema de ideas y representaciones sobre el mundo circundante y está ligado a diferentes campos del conocimiento; se relaciona con significados asignados desde las ciencias sociales, humanas, económicas, políticas, de salud, jurídicas y forenses y las administrativas, también en las artes y la ética; igualmente aparece en el lenguaje científico y en el académico y está unido al movimiento social de mujeres, al debate teórico-feminista $y$ es ampliamente empleado en el lenguaje común y cotidiano ${ }^{(2,9)}$. 
Tabla 1. Elementos del análisis conceptual de violencia de género con el método evolutivo de Rodgers

\begin{tabular}{|c|c|c|}
\hline Elementos analizados & Aspecto a destacar & Estudios \\
\hline Antecedentes & $\begin{array}{l}\text { Representan las situaciones, eventos o fenómenos que preceden } \\
\text { a un concepto de interés }\end{array}$ & $\begin{array}{l}\text { Abreu }^{6} \text { Pérez }^{13} \text {, Fernández } \\
\text { Gómez } \\
\text { Góm }^{25}, \text { Castro }^{26}, \text { Santos }^{27} \text {. }\end{array}$ \\
\hline Consecuencias & $\begin{array}{l}\text { Corresponden a los resultados del empleo del fenómeno } \\
\text { analizado }\end{array}$ & $\begin{array}{l}\text { Consejería Pres. Equid. Muj. 4, Gómez }{ }^{25} \text {, } \\
\text { Santos }^{27} \text {, ONU 28, Garcia-Moreno }{ }^{29} \text {, } \\
\text { Lafaurie }^{30-32} \text {, Marioly } 31 \text {, Álvarez }{ }^{33} \text {. }\end{array}$ \\
\hline $\begin{array}{l}\text { Términos subrogados o } \\
\text { substitutos }\end{array}$ & $\begin{array}{l}\text { Diversos términos, palabras o expresiones utilizadas con el } \\
\text { mismo sentido del concepto }\end{array}$ & $\begin{array}{l}\text { Razera 21, García-Moreno } 29 \text {, Casique } 35 \text {, } \\
\text { Domínguez } 36 \text {, Quintana } 37 \text {, Solizko } 38\end{array}$ \\
\hline $\begin{array}{l}\text { Conceptos } \\
\text { relacionados }\end{array}$ & $\begin{array}{l}\text { Conceptos relacionados, que no definen en su totalidad el } \\
\text { concepto en discusión }\end{array}$ & Añon 2 , Peris 12, Pérez ${ }^{13}$, Blanco \\
\hline Atributos & $\begin{array}{l}\text { Definición real del término, así como las expresiones o palabras } \\
\text { utilizadas para representar los fenómenos. }\end{array}$ & $\begin{array}{l}\text { Abreu6, Guzmán y Montaño15, Ramírez y } \\
\text { Ariza }^{20} \text {. }\end{array}$ \\
\hline
\end{tabular}

La violencia de género se produce en un marco de desigualdad, no se refiere exclusivamente a las mujeres, también puede ser experimentada por hombres y personas de diferente identidad de género, refleja la asimetría existente en las relaciones de poder entre hombres y mujeres $(6,10,11)$. Corresponde a una violencia estructural, que se sostiene en el marco de una cultura edificada sobre la lógica de la dominación y las relaciones de poder naturalizadas que hacen aparecer al sometimiento y la inferioridad de las mujeres como hechos normales y que invisibilizan las diferencias y otorgan un valor distinto a cada una de las identidades ${ }^{(12,13)}$. Se considera que es un dispositivo político-cultural de dominación, que vulnera los derechos humanos y la ciudadanía (14).

\section{Atributos o características}

Las diversas disciplinas definen la violencia de género de acuerdo a sus supuestos teóricos y metodológicos, sin embargo, el fenómeno es descrito con base en las diferentes formas de discriminación y condiciones de desigualdad cuyo común denominador es el poder que se expresa desde el sistema patriarcal en la dominación masculina(15).

Es un concepto complejo, los atributos se organizan en dos grupos, uno corresponde al carácter de la violencia y el otro agrupa las diversas expresiones de la misma.

El carácter de la violencia de género es estructural y relacional, la violencia está inmersa en la estructura social donde se mantiene la subordinación de la mujer(16). Es un ejercicio que produce daño a quien la recibe y se expresa en el marco de las relaciones asimétricas de poder entre hombres y mujeres"(17); incluye a personas de diferente identidad de género y preferencia sexual"(18).

El carácter relacional implica opresión y el deseo de dominación que se relaciona con la violación de los derechos humanos, inequidad, exclusión, discriminación, control, que se dan un contexto determinado $(6,19,20)$.

Respecto a las expresiones, estas son variadas, se dan tanto en el ámbito público, como en el privado; ejemplos de ellas son, entre otras, todas las formas de discriminación hacia la mujer en distintos niveles (político, institucional, laboral), el acoso sexual, la violación, el tráfico para prostitución, la utilización del cuerpo femenino como objeto de consumo; todas las formas de maltrato físico, psicológico, social y sexual en cualquier contexto, incluido el marco del conflicto armado; formas de violencia que ocasionan una escala de daños que pueden culminar en la muerte ${ }^{(21-24)}$.

\section{Antecedentes}

Los antecedentes de la violencia de género están ligados al sistema de dominación de las mujeres denominado patriarcado; el cual responde a relaciones de dominación social y política, sus raíces son sociales e históricas donde la autoridad del hombre es determinante y la mujer está ubicada en una posición de subordinación y de exclusión en diferentes ámbitos; su vivencia es distinta dependiendo de la raza, la posición social, la edad, el origen geográfico entre otras dimensiones y comparte rasgos generales como la violencia contra las mujeres, la cosificación de las mujeres y la asociación de lo femenino con lo privado y de lo masculino con lo público, adicional a lo anterior le 
otorga a las mujeres un estatus simbólico menor que legitima el uso de la violencia contra ella $(6,13,25,26)$.

De este sistema se derivan situaciones de desigualdad de oportunidades, el acceso desigual a los recursos y servicios de justicia, servicios públicos, a la discriminación laboral y salarial lo cual ha sido señalado por organismos defensores de los derechos humanos(16,17,27).

\section{Consecuencias}

Las consecuencias de la violencia de género incluyen repercusiones para el desarrollo económico, político, social y cultural de las sociedades, así mismo la exclusión y fragmentación de los derechos, la reducción de la participación política y la minimización de las mujeres como sujeto político(25).

Se presentan consecuencias como la restricción, coacción o la privación arbitraria de la libertad, la limitación de las capacidades y las oportunidades tanto en el ámbito público o en el privado; genera en las personas daño o sufrimiento físico, mental, sexual, psicológico, económico o patrimonial $(9,27,28)$.

En quienes experimentan violencia de género es posible identificar consecuencias relacionadas con la alteración de la salud que generan mayores demandas a los servicios de atención ambulatoria en salud y de atención en salas de emergencia; a nivel físico se presentan situaciones como traumatismos, fracturas, heridas, quemaduras, contusiones, hemorragias, hematomas, infecciones de vías urinarias a repetición, dolores pélvicos crónicos, cefalea, síndrome de colon irritable, mialgias, entre otras; también se presentan consecuencias a nivel psicológico y de conducta como depresión, ansiedad, disminución de la autoestima, sentimientos de culpa, vergüenza, trastornos psicosomático, de estrés postraumáticos y de la alimentación, crisis de pánico, fobias, conductas sexuales de riesgo, abuso de alcohol, drogas, tabaco, y escaso cuidado personal(2933).

\section{Términos relacionados}

Se encontraron diferentes términos en las diversas áreas del conocimiento al referirse a violencia de género, entre los términos usados están el de violencia estructural, violencia patriarcal; también términos como violencia común(12,13,26). La violencia de género se relaciona con términos como desigualdad de género, inequidad de género, exclusión, control, dominación, relaciones asimétricas. También la violencia de género está relacionada con efectos en el orden público, el conflicto armado y decisiones jurídicas $(2,13)$.

\section{Términos subrogados o sustitutos}

El término violencia de género fue acuñado en la década del 90 y se ha consolidado desde entonces, fundamentalmente a través de instrumentos internacionales y regionales de Derechos Humanos. Las aproximaciones provistas por la Conferencia Mundial de Derechos Humanos, celebrada por las Naciones Unidas en Viena en 1993; la Declaración sobre la eliminación de la violencia contra la mujer, del mismo año (ONU 1993); la Convención Interamericana para Prevenir, Sancionar y Erradicar la Violencia contra la Mujer (OEA 72 1994) y la Conferencia Mundial sobre la Mujer en Beijing (ONU 1995); ninguno de estos documentos aborda la violencia de género como fenómeno, sino que se enfocan en un tipo particular de ella: la violencia contra las mujeres( ${ }^{(4)}$.

Una expresión usada para referirse a la violencia de género es la violencia de pareja íntima - VPI que puede ocurrir en toda clase de relaciones íntimas, como el matrimonio, la unión libre, las relaciones acordadas entre parejas de sexo opuesto o del mismo sexo, entre adultos y entre adolescentes; violencia que puede continuar aún si la relación ha terminado; en donde lo que prevalece en la dinámica de esas relaciones es una asimetría de los géneros que se presenta como una manifestación producida y reproducida por estructuras sociales de dominación y que es reforzada por la ideología patriarcal $(21,34,35)$.

Los términos violencia de pareja, violencia contra la mujer, violencia sexista, violencia sexual, violencia en el noviazgo, son términos sustitutos, así mismo, violencia doméstica es otro término relacionado, es la que se experimenta al interior del espacio doméstico, aunque los actores involucrados no tengan relaciones de parentesco entre sí. Otro término que se encontró es el de violencia intrafamiliar que se asocia a personas relacionadas por un vínculo de parentesco, independientemente del espacio físico donde tiene lugar(29,36-38).

\section{Discusión}

La violencia de género es un reflejo de las desigualdades e inequidades de género que se presenta en todos los espacios e interacciones entre 
las personas. La mantiene y perpetúa la estructura de poder que se ejerce sobre las mujeres y las relaciones jerárquicas entre los géneros ${ }^{(39)}$. De acuerdo con Castro "la noción de violencia estructural hace referencia a una lógica que produce y reproduce la violencia, y que es constitutivo de la propia estructura social(26).

Esta violencia es un fenómeno social universal con diversas expresiones o manifestaciones; el concepto lo ha usado el movimiento social de mujeres y la literatura feminista como una categoría que permite diferenciar aspectos conceptuales. La comprensión de violencia de género como una condición de subordinación de las mujeres, hace necesaria la implementación de políticas públicas que visibilicen el fenómeno con todas sus características y que además tenga en cuenta el contexto en el que se produce $^{(14) .}$

Nos adherimos a la siguiente definición: La violencia de género es de carácter estructural, social, político y relacional, constituye una violación a los derechos humanos, rompe el derecho a la vida, la dignidad, la integridad física y moral, la igualdad, la seguridad, la libertad, la autonomía y el respeto; es un fenómeno social de múltiples y diversas dimensiones forjado por un sistema patriarcal que da lugar a la subordinación estructural de las mujeres y cuya consecuencia repercute en el desarrollo humano, social y político, en la expresión de las potencialidades y habilidades de los seres humanos; conlleva a limitaciones funcionales, trastornos mentales, mayor número de visitas a las instituciones de salud y perpetúa las relaciones de poder que minimizan el papel y el estatus de la mujer en la sociedad en ámbitos como la familia, la escuela y el trabajo entre otros, determinadas por condiciones socioculturales, históricas y políticas concretas $(40,41)$.

La claridad en los usos del concepto y en el de los diferentes términos empleados conexamente como relacionados o subrogados, aporta a la transformación de los procesos que tienen lugar en la aplicación de los diferentes campos del conocimiento entre ellos de las humanidades, las ciencias sociales, la educación y la salud, como también en el ejercicio de una práctica con perspectiva de género en los diversos campos de actuación, entre ellos en la salud, justicia, economía, en lo social, etc., también en el papel que juegan los medios de comunicación y tendrá efecto en el marco normativo, la investigación futura y en la toma de decisiones(42).

En el campo de la salud ayuda a considerar la vulnerabilidad social de las mujeres, las niñas, las adolescentes y las adultas mayores en las estrategias de promoción de la salud, la prevención y erradicación de la violencia de género y en las medidas de atención que se adopten y de manera particular en la atención en salud en los diferentes niveles especialmente en el nivel comunitario, en las áreas de atención de emergencia y en los programas de salud mental, salud de la mujer y de atención prenatal $(30,43,44)$.

La claridad en el concepto aportará en la formación del personal de salud, particularmente en la de médicos y enfermeras en los campos de salud familiar, ginecología y obstetricia, salud mental, enfermedades crónicas, en promoción de la salud y gerencia en salud, también en la formación de otras profesiones como trabajo social, psicología, antropología, sociología, derecho, administración y economía(26,45).

Al comprender el concepto de violencia de género se podrá estudiar y proponer las estrategias y maneras para una práctica en salud de calidad en los diferentes niveles de atención, implementar intervenciones sociales, contribuir al desarrollo de la investigación en este tópico y a la formación de profesionales en todos los campos del conocimiento. Se recomienda que se realicen estudios en esta temática en el marco de la interseccionalidad en los cuales se tenga en cuenta la posición social, el género, la raza, la etnicidad, la edad, la orientación sexual, la condición de discapacidad y la religión, los cuales contribuyen a comprender los fenómenos de violencia como un fenómeno social y a entender que los grupos no son homogéneos y que se presentan interacciones entre ellos( $(42,46,47)$.

\section{Conclusiones}

La violencia de género es un fenómeno de carácter estructural, social, político y relacional, constituye una violación a los derechos humanos, afecta principalmente a las mujeres, no excluye a personas con identidades de género diversas, rompe el derecho a la vida, la dignidad, la integridad física y moral, la igualdad, la seguridad, la libertad, la autonomía y el respeto. 
Conflicto de intereses: No se recibió apoyo financiero para la escritura de este manuscrito, este manuscrito está basado en la revisión de literatura, por lo cual no hay involucramiento de muestras, ni de personas por lo que no se requirió aval de comité de ética.

\section{Referencias}

1. Walker L, Avant K. Strategies for Theory Construction in Nursing. 4th ed. New Jersey; 2005.

2. Añon MJ. Violencia con género. A propósito del concepto y la concepción de la violencia contra las mujeres. Cuadernos Electrónicos de Filosofía del Derecho [Internet]. 2016 Jun [citado 2019 Mar]; 33(2016) 1-26. Disponible en: https://ojs.uv.es/index.php/CEFD/article/view/8257/803 5

3. Congreso de la República 2008. Ley 1257 de 2008. Congreso de la República. Bogotá, Colombia. [Internet]. Disponible en: http://www.secretariasenado.gov.co/senado/basedoc/ley_ 1257_2008.html

4. Alta Consejería Presidencial para la Equidad de la Mujer. Lineamientos de la política pública nacional de equidad de género para las mujeres. 2012;117.

5. Peris M. La importancia de la terminología en la conceptualización de la violencia de género. Oñati Sociolegal Ser. 2015;5(2):716-44.

6. Abreu M. La violencia de género entre el concepto jurídico y la realidad social. Rev Electrónica Cienc Penal y Criminol. 2006; 02:1-13.

7. Tofthagen R, Fagerstrøm LM. Rodgers evolutionary concept analysis a valid method for developing knowledge in nursing science. Scand J Caring Sci. 2010;24(1):21-31.

8. Rodgers B, Knafl K. Concept development in nursing: foundations, techniques, and applications. 2nd ed. W.B. Saunders P, editor. London; 2000.

9. Pacheco M. La violencia: conceptualización y elementos para su estudio. Política y Cult. 2016;(46):7-31.

10. Bodelón, E. Violencia institucional y violencia de género. Anales de la cátedra Francisco Suárez, 2015; 48, 131-155.

11. Herrera M del C. Análisis Psicosocial de la violencia de género: sexismo, poder $\mathrm{y}$ amenaza como factores explicativos. Universidad de Granada; 2009.

12. Peris M. La despolitización de la violencia de género a través de la terminología. Asparkía. 2013; 24:176-93.

13. Pérez M. El concepto de "violencia de género" como espejismo hermenéutico. Igualdad, autonomía personal y derechos sociales; 2018;8:69-88.

14. Rico N. Violencia de género: un problema de Derechos Humanos. Vol. 16, Serie mujer y desarrollo. 1996.

15. Guzmán Barcos V, Montaño Virreira S. Políticas públicas e institucionalidad de género en América Latina (1985-2010). CEPAL-Serie mujer y Desarro. 2012;(118):39.

16. Fernández $\mathrm{S}$. La violencia de género en las prácticas institucionales de salud: afectaciones del derecho a la salud y a las condiciones de trabajo en salud. Gerencia, Política y Salud. 2007;6(12):52-76.

17. Barreto M. Violencia De Género Y Denuncia Pública En La Universidad. Rev Mex Sociol. 2017;79(2):261-86.

18. Arisó 0 , Mérida R. Los géneros de la violencia Una reflexión queer sobre la «violencia de género». Egales, editor. 2010. $181 \mathrm{p}$.
19. Gómez C, Murad R, Calderón MC, De Miguel A, Tobergte DR, Curtis $\mathrm{S}$, et al. Informe mundial sobre la violencia y la salud. Oms. 2013;18(resumen tratado de ginebra):547.

20. Ramírez-Rodríguez María Himelda, Ariza-Sosa Gladys Rocío. Lo político de la violencia en las relaciones de pareja como problema de salud pública. rev.fac.med. 2015; 63( 3 ): 517 525. Available from: http://www.scielo.org.co/scielo.php?script=sci_arttext\&pid =S0120-00112015000300020\&lng=en. http://dx.doi.org/10.15446/revfacmed.v63n3.45191.

21. Razera, Josiane, Gaspodini, Icaro Bonamigo y Falcke, Denise. Violencia de pareja íntima y género A / simetría: una revisión bibliográfica integradora. Psico-USF. 2017; 22 (3), 401-412

22. Puente-Martínez Alicia, Ubillos-Landa Silvia, Echeburúa Enrique, Páez-Rovira Darío. Factores de riesgo asociados a la violencia sufrida por la mujer en la pareja: una revisión de meta-análisis y estudios recientes. Anal. Psicol. 2016; 32(1): 295-306. Disponible en: http://scielo.isciii.es/scielo.php?script=sci_arttext\&pid=S02 12-97282016000100034\&lng=es. http://dx.doi.org/10.6018/analesps.32.1.189161

23. Femenías ML, Soza Rossi P. Poder y violencia sobre el cuerpo de las mujeres. Sociologias. 2009;11(21):42-65.

24. Munevar DI. De las violencias que encierran a las acciones que sanan. 1st ed. Colombia UN de, editor. Bogotá, Colombia; 2011. $440 \mathrm{p}$.

25. Gómez D, Pineda J. Desarrollo económico local en clave de género. Doc Política. 2018;(8):1-23.

26. Castro R. Violencia de género. In: Conceptos clave en los estudios de género. Ciudad de México; 2016. p. 19.

27. Santos Calderon JM. Derechos de las mujeres: Principales instrumentos y normas internacionales y nacionales. Colección Cuadernos Legis y pueblos indígenas Colomb. 2013; 6:9.

28. Organización de las Naciones Unidas. Declaración de las Naciones Unidas sobre la Violencia contra la Mujer. [Internet]. Nueva York (EU): ONU; 1993 Dic. Disponible en: https://www.ohchr.org/sp/professionalinterest/pages/viol enceagainstwomen.aspx

29. Organización de las Naciones Unidas. Estudio multipaís de la OMS sobre salud de la mujer y violencia doméstica contra la mujer. [Internet]. Nueva York (EU): ONU; 2005. Disponible

en: https://www.who.int/gender/violence/who_multicountry_ study/summary_report/chapter1/es/

30. Lafaurie MM, Veloza E. Nada justifica la violencia contra las mujeres. Trazando una ruta para motivar reflexiones en torno a las violencias basadas en género, Bogotá: OIMMinisterio de la Protección Social; 2010.

31. Marioly RH, Laura LA, Yoan HC, Eduardo CÁ, Yisel ÁR. Caracterización de las mujeres maltratadas por su pareja desde la perspectiva de género. MediSur. 2013;15-26.

32. Lafaurie M. ¿Por qué pensar en la salud de las mujeres? Rev Salud Bosque. 2016;1(1):79-90.

33. Álvarez López, Esther, Brito, Carolina, Arbach, Karin, Pueyo, Antonio Andrés, Detección de la Violencia contra la Pareja por Profesionales de la Salud. Anuario de Psicología Jurídica. 2010; 20:31-41.

34. Blanco Pilar, Ruiz-Jarabo Consuelo, García de Vinuesa Leonor, Martín-García Mar. La violencia de pareja y la salud de las mujeres. Gac Sanit. 2004; 18(4): 182-188. Disponible en: 
http://scielo.isciii.es/scielo.php?script=sci_arttext\&pid=S02 13-91112004000400029\&lng=es.

35. Casique Casique L, Furegato ARegina Ferreira. Violence against women: theoretical reflections. Rev. Latino-Am. Enfermagem [Internet]. 2006; 14(6): 950-956. Available from: http://dx.doi.org/10.1590/S010411692006000600018

36. Domínguez Fuentes, Juan Manuel, García Leiva, Patricia, Cuberos Casado, Inmaculada, Violencia contra las mujeres en el ámbito doméstico: consecuencias sobre la salud psicosocial. Anales de Psicología. 2008;24(1):115-120. Recuperado de: https://www.redalyc.org/articulo.oa?id=16724114

37. Quintana Lorena, Otegui Joselí. Una mirada al abordaje asistencial a mujeres víctimas de violencia doméstica. Rev. Méd. Urug. 2017; 33(3): 142-147. Disponible en: http://www.scielo.edu.uy/scielo.php?script=sci_arttext\&pi $\mathrm{d}=$ S1688-03902017000300142\&lng=es.

38. Solyszko Gomes, I. (2016). Y no fueron felices para siempre: Desafíos para la intervención pensando la familia y la violencia. Hojas y Hablas, (13), 133-140. Recuperado a partir http://revistas.unimonserrate.edu.co:8080/hojasyhablas/a rticle/view/89

39. Zurbano B, Vaya I, Campos B. Concepto y representación de la violencia de género. Reflexiones sobre el impacto en la población joven. Oñati Socio-Legal Series. 2015;5(2):82245.

40. Fernández S, Hernández G, Paniagua R. Violencia de género. 1st ed. Universidad de Antioquia, editor. Violencia de género en la Universidad de Antioquia. ASOPRUDEA; 2007. 401 p.

41. Castañeda MP, Torres P. Cotidiano. Otros debates sobre la violencia Concepciones sobre la violencia: una mirada antropológica. 2015;7-21.

42. Guzmán Ordaz R, Jiménez Rodrigo LM. La interseccionalidad como instrumento analítico de interpelación en la violencia de género. Oñati Socio-Legal Series. 2015;5(2):596-612.

43. Lafaurie-Villamil MM, Cuadros-Salazar LL, García-Bocanegra PA, Hernández-Ayala IJ, Pulido-Espinosa JS, Reynales-Triana LF, Rodríguez-Díaz AP, Sánchez-Cortes PA, Siatoya-Pinto LA, Torres-Rivera MA. Violencia de la pareja íntima durante el embarazo y sus repercusiones en la salud mental. Rev. Cienc. Cuidado. 2015;12(2):100-16. Disponible en: https://revistas.ufps.edu.co/index.php/cienciaycuidado/art icle/view/512.

44. Amurrio M, Larrinaga A, Usategui E, Del Valle A. Violencia de género en las relaciones de pareja de adolescentes y jóvenes de Bilbao. Gizarte zerbitzuetarako aldizkaria = Revista de servicios sociales [Internet]. 2010 Jun [citado 2018 Mar]; 47:121-134. Disponible en: https://dialnet.unirioja.es/servlet/articulo?codigo=326282 1

45. Gómez C, Murad R, Calderón M. Historias de violencia, roles, prácticas y discursos legitimadores. Violencia contra las mujeres en Colombia 2000-2010. J Chem Inf Model. 2013;53(9):1689-99.

46. González Lozoya I, Serrano Martínez A, García Sánchez N, Del Campo Giménez M, Moreno Ruiz B, González Lozoya AB, et al. Conocimientos sobre violencia de género de la población que consulta en Atención. Aten Primaria. 2011;43(9):459-64.

47. Cruz STM da, Espíndula DHP, Trindade ZA. Violência de Gênero e seus Autores: Representações dos Profissionais de Saúde. Psico-USF. 2017;22(3):555-67. 